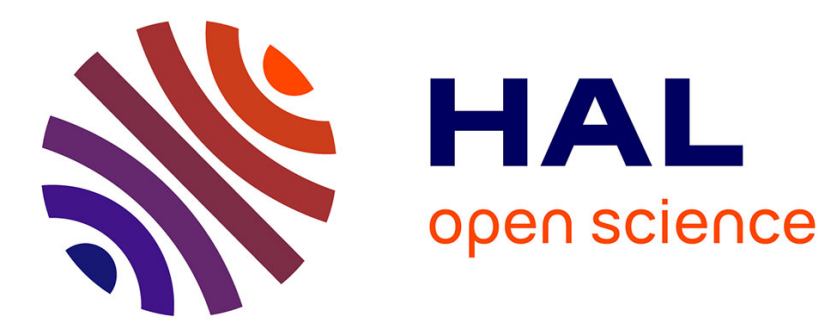

\title{
Deformation banding and its influence on deformation textures formation
}

C. Lee, B. Duggan, R. Smallman

\section{To cite this version:}

C. Lee, B. Duggan, R. Smallman. Deformation banding and its influence on deformation textures formation. Journal de Physique IV Proceedings, 1993, 03 (C7), pp.C7-2027-C7-2032. 10.1051/jp4:19937323 . jpa-00251969

\section{HAL Id: jpa-00251969 https://hal.science/jpa-00251969}

Submitted on 1 Jan 1993

HAL is a multi-disciplinary open access archive for the deposit and dissemination of scientific research documents, whether they are published or not. The documents may come from teaching and research institutions in France or abroad, or from public or private research centers.
L'archive ouverte pluridisciplinaire HAL, est destinée au dépôt et à la diffusion de documents scientifiques de niveau recherche, publiés ou non, émanant des établissements d'enseignement et de recherche français ou étrangers, des laboratoires publics ou privés. 


\title{
Deformation banding and its influence on deformation textures formation
}

\author{
C.S. LEE, B.J. DUGGAN* and R.E. SMALLMAN \\ School of Metallurgy and Materials, University of Birmingham, Birmingham B15 2TT, U.K. \\ * Mechanical Engineering Department, University of Hong Kong, Pokfulam Road, Hong Kong
}

\begin{abstract}
It is shown by a crystallographic etching technique that deformation banding is an important deformation mode in copper. In a cold rolled coarse grain copper, deformation banding forms in a three dimensional manner dividing grains into a large number of bands of different orientations. The influence of this important, but long ignored deformation mode, is studied by incorporating it into the Taylor model. The predicted textures from the new model are better than those from other existing models in mainly two aspects. Firstly, the present model predicts the co-existence of the three major FCC rolling texture components, namely $\{123\}<634\rangle$ or $S$ component, $\{112\}<111\rangle$ or $C$ and $\{110\}<112\rangle$ or $B$. The existing models are deficient in that they predict either $C$ and $S$ or $B$, but not their co-existence. The second point is that textures predicted by the existing models are always too sharp compared to the experimental textures. The deformation banding model predicts texture peaks with larger spread and hence more realistic texture
\end{abstract} sharpness.

Introduction

Deformation banding is a process in which different regions of a deforming crystal gradually rotate to different orientations as deformation proceeds. Its importance to the deformation process has been repeatedly emphasized by Barrett et. al. [1-3] and Chin [4]. However, only a limited amount of work has been done recently [5-8] mainly on single crystals or for recrystallisation studies. The purpose of the present work has been to investigate the influence of this deformation mode in polycrystalline materials.

Experimental Results and Discussions

High purity copper $(99.99 \%)$ of two different grain sizes, 40 and $3000 \mu \mathrm{m}$, with weak initial textures were cold rolled to various strains up to $92 \%$ reduction. The behaviour of the two materials has been confirmed to be typical by various standard techniques including $\mathrm{X}-\mathrm{ray}$ diffraction, optical and transmission electron microscopy. The results shown in this paper were obtained by a crystallographic etching technique recently developed by Köhlhoff et al. [9]. By etching electropolished surfaces of copper in concentrated nitric acid, the $\{111\}$ planes within grains are preferentially exposed. As a result, different etching patterns are observed in grains of different orientations.

The general cold rolled microstructure as revealed by the 
crystallographic etch is shown in figure 1. Figure 1a shows a longitudinal section of the coarse grained copper after $85 \%$ reduction. The most remarkable feature is the unexpected thickness of the layered structure. The thickness of the layers each with different orientations ranges from about 1 to $50 \mu \mathrm{m}$ and has an average value of $17.2 \mu \mathrm{m}$ (measured by a linear intercept method at 4 different locations in two specimens containing over 100 layers). This value is much smaller than the average grain thickness after $85 \%$ reduction, which is about $3000 \times 0.15$ or $450 \mu \mathrm{m}$. It therefore follows that each grain deforms to give on the average about $26(=450 / 17.2)$ layers.
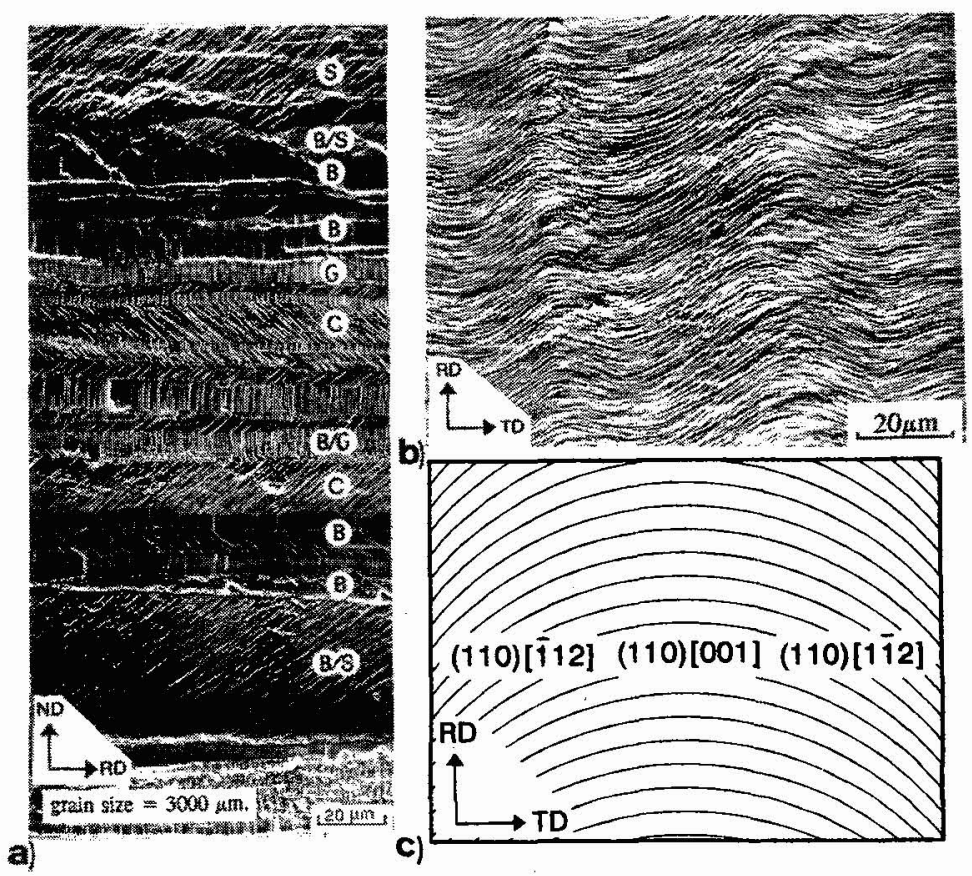

Fig. 1. a) SEM micrograph of crystallographically etched longitudinal section of coarse grained copper after $85 \%$ cold rolling; b) Rolling plane section of coarse grained copper after $70 \%$ reduction. The grain splits into alternative bands of complementary B orientations (i.e. (110) [112] and (110)[112]); c) Schematic diagram of the etching patterns of (110) $[1 \overline{1} 2],(110)[\overline{1} 12]$ AND (110) [001] when observed in the rolling plane.

Possible deformation processes which would subdivide a grain into regions of different orientation, include mechanical twinning, martensitic transformation and deformation banding. The operation of the first two processes is excluded for two reasons. Firstly, transmission electron microscope studies have not revealed any evidence of twinning or martensitic transformation in copper deformed at room temperature. Furthermore, both processes would produce lattice regions of new orientation having sharp boundaries with the matrix. Both diffuse and sharp boundaries are observed in the present studies. Diffuse boundaries are more common at low strains ( $\angle 50 \%$ reduction) and sharp boundaries at high strains ( $>85 \%$ reduction). It is therefore believed that deformation banding is responsible for the observed subdivision of the grains.

Figure $1 \mathrm{~b}$ is a micrograph taken from the rolling plane section of the coarse grained copper after $75 \%$ reduction. It clearly shows that a grain is divided into bands of two symmetrically related $\{110\}<112>$ 
orientations (i.e. (110) [112] and (110) [ $\overline{1} 12]$ ) with (110) [001] in between, fig. 1c). The average band width in the rolling plane section is measured to be $120 \mu \mathrm{m}$. This is again much narrower than the original grain width of $3000 \mu \mathrm{m}$, suggesting that on the average each grain is divided into 25 $(=3000 / 120)$ regions by banding with boundaries parallel to the longitudinal planes. It therefore appears that banding of at least two different geometries is operating simultaneously, namely banding with planes parallel to the rolling and longitudinal planes respectively. As a consequence, grains are three dimensionally subdivided and on the average, each grain will give over 600 (25x26, assuming that the two banding geometries are independent) bands in three dimensions.

Deformation banding was also observed in the fine grained copper but not as pronounced as in the coarse grained copper. From the measurement of the longitudinal section, each grain gives an average of 2.4 layers. This value together with the corresponding one from the coarse grained copper agrees reasonably well with a recent prediction by Lee et al. [10] that the average number of bands (in one dimension) formed in a grain is proportional to the square root of the grain size. No measurements have been carried out on the rolling plane of the fine grained copper because satisfactory etching cannot be achieved due to the small layer thickness in the normal direction.

Because of these important microstructural changes, it is likely that deformation banding also significantly affects the development of the deformation texture. It has been believed for many years that copper deformed at room temperature does so mainly by homogeneous slip processes. Deformation models based on homogeneous slip have indeed predicted deformation textures similar to those observed experimentally [11-12]. However, these predicted textures have a major difference from the experimentaliy determined textures.

The $\{110\}<112>$ orientation is one of the major stable rolling texture component in copper but is predicted by neither the Full constraint (FC) nor the Relaxed Constraint (RC) Taylor models [13]. Calculation with these models shows that $\{110\}<112\rangle$ is stable only if shear in the rolling plane is allowed ( $\mathrm{fig}$. 2a). However, relaxing this shear constraint in the deformation not only produces a severe incompatible shape change with the surrounding, but also destroys other experimentally observed stable texture components, namely $\{123\}<634>$ and $\{112\}<111>$. An earlier analysis by Aernoudt and stüwe [14] affords a possible solution in suggesting that

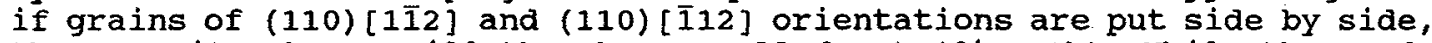
the opposite shears will then be cancelled out (fig. $2 \mathrm{~b}$ ). While they made no further suggestion on how the special microstructural arrangement forms, it is clear from figure 2 that this structure which stabilises the $\{110\}<112>$ orientation could be produced by deformation banding.

\section{Modelling of Deformation banding}

It is clear from the above discussion that the deficiency of the current deformation models is mainly due to their failure to address the significance of deformation banding. In order to study its influence on the deformation processes, a texture simulation model (DB model) is developed by incorporating deformation banding into the Taylor model. This is done by assuming that all grains behave macroscopically as "Taylor grains", whether deformation banding occurs or not. In the original Taylor model, it is assumed that the strain in each grain equals the macroscopic external strain. If deformation banding occurs, this assumption becomes: The average strain over different regions of a banded grain should equal the macroscopic external strain. Thus, the microscopic deformation within each segment can be different from the macroscopic one, so long as their average strain is compatible to themselves and 
their surroundings.

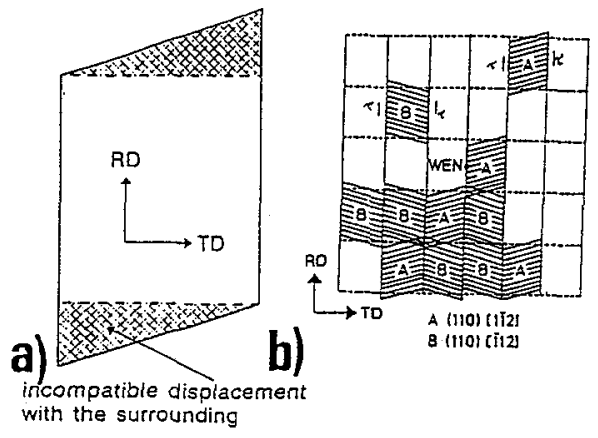

Figure 2
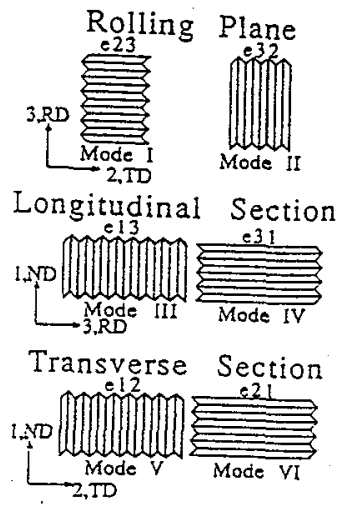

Figure 3

Fig. 2. a) The $\{110\}<112>$ texture component is stable in the Taylor type models only if it is allowed to shear freely parallel to the rolling plane. However, this results in a severe strain compatibility problem with the surrounding; b) A schematic diagram showing how the compatibility problem can be solved by putting symmetrically oriented $\{110\}<112\rangle$ volumes next to each other such that the opposite shears produced in the two components cancel each other out (after Aernoudt and Stijwe, 1970).

Fig. 3. A schematic diagram showing six geometrically possible modes of deformation banding in rolled grain.

From a purely geometrical point of view, there can be six different modes of deformation banding (fig. 3). However, only three banding modes II, IV and VI, which are consistent with the banding geometries observed experimentally, need further consideration.

With the three banding modes operating simultaneously, the three shear strain components $\left(\epsilon_{31}, \epsilon_{21}\right.$ and $\left.\epsilon_{32}\right)$ of grains are no longer constrained to zero. They are partly relaxed. The term partly is used because the relaxation is not as free as it is assumed in the RC model. In the RC model, the shear strain $\epsilon_{31}$ and $\epsilon_{21}$ are relaxed without any further restriction such as the geometric constraint from the neighbouring grains. In real materials when deformation banding take place, these shear strains are in fact partly relaxed with constraints from the neighbouring bands. There are two types of constraints, the first requires that the average shear strains over the grain are zero; the second type requires continuity of displacement across band boundaries. Within these restrictions, the shear strains can be any value. Roughly speaking, the material's behaviour is in-between the description by the FC and the RC model.

However, modelling of the simultaneous operating of the three banding modes is still not possible due to the mathematical complexity. Instead, a one dimensional model, in which only one banding mode is considered, is used to estimate the possible effect of banding. The influence of the remaining two banding modes are approximated by some simplification processes. As the main effect of the banding is to allow a partial relaxation of the shear, it is natural to approximate its effect by either fully relax or fully constraint the shears. These correspond to the RC and the FC model respectively. Either of the approximation is acceptable as both the RC and the FC models predict textures similar to the experimentally obtained copper type texture. However, as the quality 
of the textures predicted by the RC model are generally considered better, the former approximation was hence used.

The remaining question is which of the two modes should be approximated. A sensible choice is to consider the two modes which approximation by freely relaxing the corresponding shears produce least error in the predicted texture. From the results of the classical RC model, the two modes corresponding to the relaxation of $\epsilon_{31}$ and $\epsilon_{21}$ should be used. It is because freely relaxing $\epsilon_{32}$ not only produces a larger incompatible deformation, but also destroys the other stable texture component ( $S$ and $C$ ) in copper. Thus only mode II banding is modelled and mode IV and VI are approximated by freely relaxing the corresponding shear $\epsilon_{31}$ and $\epsilon_{21}$ as in the classical RC model.

With these approximations, the one dimensional model would suffer from some strain compatibility problems as in the RC model. $\epsilon_{31}$ and $\epsilon_{21}$ might not be continuous across the band boundaries; as they are generally not continuous across the grain boundaries in the RC model. In the RC model, it is suggested that these incompatibilies do not cause severe error so long as the grain thickness is small compared to the other two dimensions. Concerning this, it should be pointed out that the individual band in a banded grain has in fact a more favourable geometry for relaxation than a non-banded grain in the RC model. As the compatibility problem does not cause significant error in the predictions of the RC model, it is unlikely that it will in the DB model. Perhaps the best justification of ignoring this problem in the present one dimensional deformation banding model is that the simulated textures are closer to the experimental textures.

A one dimensional model based on mode II banding is then formulated with the following rules:

$I$. The average strain over a grain equals the macroscopic strain:

$$
\begin{aligned}
& \epsilon_{32}^{(1)} x^{(1)}+\epsilon_{32}^{(2)} x^{(2)}=0 \text { with } x^{(1)}+x^{(2)}=1 ; \quad x=\text { volume fraction; } \\
& \epsilon_{11}^{(j)}=e_{11} ; \quad \epsilon_{22}^{(j)}=0 ; \quad \epsilon_{33}^{(j)}=-e_{11} ; \text { and } \epsilon_{21}^{(j)} \text { and } \epsilon_{31}^{(j)} \text { relaxed; }
\end{aligned}
$$

where the bracketed superscripts refer to the deformation band number. II. Total internal work done, $\mathrm{W}_{D B}$, is minimum:

ie. $W_{D B}=x^{(1)} \sum \gamma_{i}^{(1)} \tau_{\mathfrak{i}}+x^{(2)} \sum \gamma_{i}^{(2)} \tau_{\mathfrak{i}}$ is minimized by selecting suitable slip systems which can accommodate the strain in $I_{i}$ where $\gamma_{i}$ and $\tau_{i}$ are respectively the shear strain and CRSS of on the ith slip system.

III. The DB model is used only if the deformation energy is significantly smaller than that required by the $\mathrm{RC}$ model (ie. $W_{D B}<0.95 \mathrm{~W}_{\mathrm{RC}}$ ); otherwise, the RC (pancake) model is used.

$\gamma_{i}$ and crystal rotations can then be solved with the above rules. Rolling textures are simulated in steps of 0.05 true strain up to $92 \%$ reduction with 100 grains of random orientations as the starting material.

Simulation Results and Discussions

Only simulated textures from the DB and the RC (pancake) model after 87\% reductions are reported here. Full account of the simulated textures at other strains and by other models (eg. FC and Sachs) are reported elsewhere [15-16]. It is clear from figure 4 that the copper-type rolling texture is better simulated by the DB model than the RC model both in term of positions of orientation density peaks and texture sharpness. Particularly, the B component is not predicted by the RC model; whereas, all of the three major copper texture components, $S, C$ and $B$ are predicted by the DB model. Sharpness of the texture predicted by the $D B$ 
model is lower than that by the RC model. This partly solves the well known problem that simulated textures are usually sharper than the experimental textures.

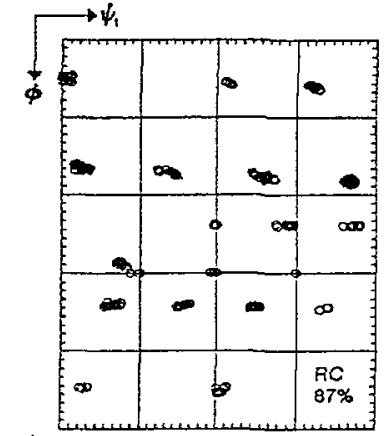

a)

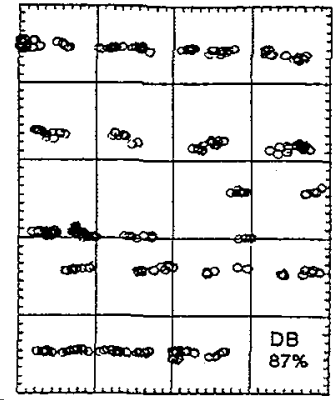

b)

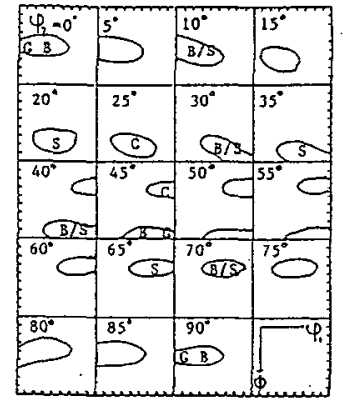

C)

Fig. 4. Simulated textures after $87 \%$ rolling by a) RC; b) DB models; c) a schematic diagram showing positions of the major fcc rolling texture components.

Conclusions

Deformation banding is shown to be an important deformation mode in copper. It is more pronounced in coarse-grained than fine-grained materials. In the coarse-grained material, different banding geometries form simultaneously, three dimensionally dividing grains into a large number of bands. The influence of deformation banding on texture development is estimated by incorporating deformation banding into the Taylor model. The simulated rolling textures from the deformation banding model are closer to the experimental textures than those from the Relaxed constraint model. In particular, the $\{110\}\langle 112\rangle$ texture component in rolled copper is shown to be formed by deformation banding using both of microstructure observations and texture simulations.

\section{Acknowledgement}

C.S. Lee gladly acknowledges the Croucher Foundation for financial support in the form of a Fellowship.

\section{REFERENCES}

1. C.S. Barrett, Trans. AIME 135 (1939) 296.

2. C.S. Barrett, and L.H. Levenson, Trans. AIME 137 (1940) 112.

3. C.S. Barrett, The structure of Metals (1st ed., McGraw-Hill, N.Y.) (1943) p.381-419.

4. G.Y. Chin, Textures in Research and Practice, Berlin (1969) 236.

5. S.P. Bellier, and R.D. Doherty, Acta Metall., 25 (1977) 521.

6. Y. Inokuti, and R.D. Doherty, Acta Metall., 26 (1978) 61.

7. K. Higashida, J. Takamura, and N. Narita, Mat. Sci. Eng., 81 (1986) 239.

8. A. Akef, and J.H. Driver, Mat. Sci. Eng., A132 (1991) 245.

9. G.D. Köhlhoff, X. Sun, and K. Lücke, Proc. 8th Int. Conf. on Textures of Materials (1988) p.183.

10. C.S. Lee, B.J. Duggan, and R.E. Smallman, Acta Metall. Mater. (accepted).

11. J.S. Kallend, and G.J. Davies, Phil. Mag., 25 (1972) 471.

12. I.L. Dillamore, and H. Katoh, H., Met. Sci., 8 (1974) 21.

13. J. Hirsch, and K. Lücke, Acta Metall. 36 (1988) 2883.

14. E. Aernoudt, and H.P. Stüwe, Z. Metallk., 61 (1970) 128.

15. C.S. Lee, and B.J. Duggan, Acta Metall. Mater. (accepted).

16. C.S. Lee, R.E. Smallman, and B.J. Duggan, to be published.

17. C.S. Lee, and B.J. Duggan, Scripta Metal1. Mater., 28 (1992) 121. 